\title{
Gallai-Ramsey numbers for a class of graphs with five vertices *
}

\author{
Xihe $\mathrm{Li}^{a, b}$ and Ligong Wang ${ }^{a, b, \dagger}$ \\ ${ }^{a}$ Department of Applied Mathematics, School of Science, \\ Northwestern Polytechnical University, Xi'an, Shaanxi 710072, P. R. China. \\ ${ }^{b}$ Xi'an-Budapest Joint Research Center for Combinatorics, \\ Northwestern Polytechnical University, Xi'an, Shaanxi 710129, P. R. China. \\ E-mail: lxhdhr@163.com; lgwangmath@163.com
}

\begin{abstract}
Given two graphs $G$ and $H$, the $k$-colored Gallai-Ramsey number $\operatorname{gr}_{k}(G: H)$ is defined to be the minimum integer $n$ such that every $k$-coloring of the complete graph on $n$ vertices contains either a rainbow copy of $G$ or a monochromatic copy of $H$. In this paper, we consider $g r_{k}\left(K_{3}: H\right)$ where $H$ is a connected graph with five vertices and at most six edges. There are in total thirteen graphs in this graph class, and the Gallai-Ramsey numbers for some of them have been studied step by step in several papers. We determine all the GallaiRamsey numbers for the remaining graphs, and we also obtain some related results for a class of unicyclic graphs.
\end{abstract}

Key Words: Gallai-Ramsey number, rainbow triangle, monochromatic subgraphs.

AMS Subject Classification (2010): 05C15, 05C55, 05D10.

\section{Introduction}

In this paper, we only consider edge-colorings of finite simple graphs. For an integer $k \geq 1$, let $c: E(G) \rightarrow[k]$ be a $k$-edge-coloring (or simply, a $k$-coloring) of a graph $G$, where $[k]:=$ $\{1,2, \ldots, k\}$. Note that $c$ is not necessarily a proper edge-coloring. A coloring of a graph is called monochromatic if all edges are colored the same, and a coloring is called rainbow if all edges are colored differently. A graph $G$ is called rainbow triangle-free if every triangle in $G$ contains at most two colors. A Gallai-k-coloring is a rainbow triangle-free coloring of a complete graph using at most $k$ colors. In 1967, Gallai [9] provided the following important structural result in his original paper, which was translated into English and endowed by comments in [14].

Theorem 1.1. ([9, 14]) In any rainbow triangle-free coloring of a complete graph, there exists a partition $V_{1}, V_{2}, \ldots, V_{m}(m \geq 2)$ of the vertices such that between the parts there are in total at most two colors, and between every pair of parts, there is only one color on the edges.

\footnotetext{
* Supported by the National Natural Science Foundation of China (No. 11871398) and the Natural Science Basic Research Plan in Shaanxi Province of China (Program No. 2018JM1032).

${ }^{\dagger}$ Corresponding author.
} 
The partition provided in Theorem 1.1 is called a Gallai-partition. Given a Gallaicoloring of a complete graph and a Gallai-partition $V_{1}, V_{2}, \ldots, V_{m}$, the subgraph induced by $\left\{v_{1}, v_{2}, \ldots, v_{m}\right\}$ is called the reduced graph of that partition, where $v_{i} \in V_{i}$ for every $i \in[m]$.

Given a graph $G$, the Ramsey number $r_{k}(G)$ is the minimum integer $n$ such that every $k$-coloring of $K_{n}$ contains a monochromatic copy of $G$. Given two graphs $G$ and $H$, the $k$ colored Gallai-Ramsey number $\operatorname{gr}_{k}(G: H)$ is defined to be the minimum integer $n$ such that every $k$-coloring of the complete graph on $n$ vertices contains either a rainbow copy of $G$ or a monochromatic copy of $H$. It is clear that $\operatorname{gr}_{k}(G: H) \leq r_{k}(H)$ for any two graphs $G$ and $H$.

Note that finding the exact values of $g_{k}(G: H)$ is far from trivial, even for some graphs $H$ with small number of vertices and edges. The Gallai-Ramsey numbers $g r_{k}\left(K_{3}: H\right)$ for all the graphs $H$ with four vertices have been found in several previous papers. However, this number for some graphs with five vertices have not been determined, and finding the exact values for these graphs is a fundamental work in this research area. In this paper, we mainly focus on $\operatorname{gr}_{k}\left(K_{3}: H\right)$ where $H$ is a connected graph with five vertices and at most six edges. There are thirteen graphs in this graph class (see Fig. 1.1), and the Gallai-Ramsey numbers for $F_{1}, F_{2}, \ldots, F_{8}$ have been studied step by step in several papers [4, 5, 10, 15, 17, 18]. We determine the Gallai-Ramsey numbers for all the remaining graphs. For more information on this topic, we refer the readers to [2, 3, 6, 11, 12, 16] and two excellent surveys [7, 8].

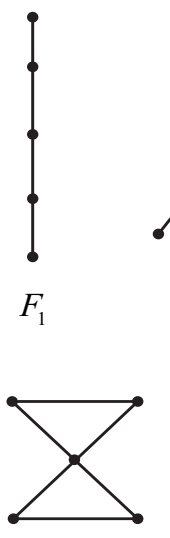

$F_{8}$

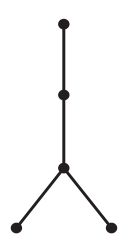

$F_{2}$

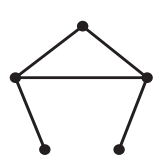

$F_{9}$
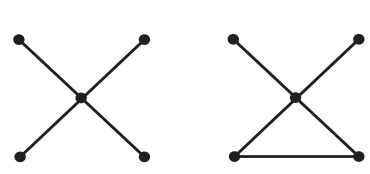

$F_{3}$

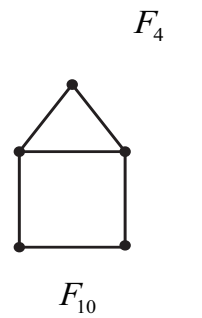

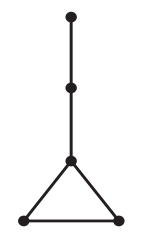

$F_{5}$

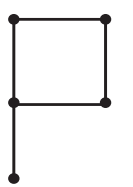

$F_{11}$
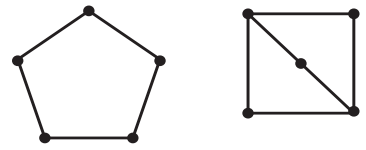

$F_{6}$
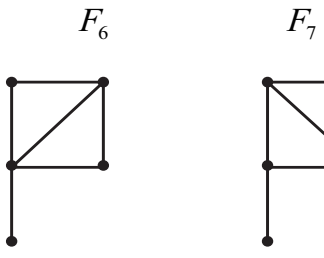

$F_{12}$

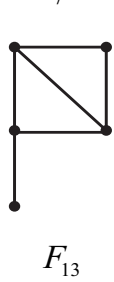

Fig. 1.1: The graphs with five vertices and at most six edges.

For graphs $F_{9}$ and $F_{10}$, we prove the following result in Section 2 ,

Theorem 1.2. For any integer $k \geq 1$,

$$
g r_{k}\left(K_{3}: F_{9}\right)=g r_{k}\left(K_{3}: F_{10}\right)= \begin{cases}8 \cdot 5^{(k-2) / 2}+1, & \text { if } k \text { is even }, \\ 4 \cdot 5^{(k-1) / 2}+1, & \text { if } k \text { is odd } .\end{cases}
$$

For graph $F_{11}$, we prove the following result in a more general form in Section 3 . In fact, we consider the Ramsey numbers and Gallai-Ramsey numbers for a class of unicyclic graph $F_{2, n}$.

Theorem 1.3. For any integer $k \geq 1, g_{k}\left(K_{3}: F_{11}\right)=k+4$.

For graphs $F_{12}$ and $F_{13}$, we prove the following result in Section 4 . 
Theorem 1.4. For any integer $k \geq 1$,

$$
\operatorname{gr}_{k}\left(K_{3}: F_{12}\right)=g r_{k}\left(K_{3}: F_{13}\right)= \begin{cases}9 \cdot 5^{(k-2) / 2}+1, & \text { if } k \text { is even, } \\ 4 \cdot 5^{(k-1) / 2}+1, & \text { if } k \text { is odd. }\end{cases}
$$

Furthermore, we define some notation and terminology. Let $c(u v)$ denote the color used on the edge $u v$. For $U, V \subseteq V(G)$ with $U \cap V=\emptyset$, let $E(U, V)$ denote the set of edges between $U$ and $V$, and $C(U, V)$ denote the set of colors used on the edges in $E(U, V)$. If $|C(U, V)|=1$, i.e., all the edges in $E(U, V)$ have a single color, then we use $c(U, V)$ to denote this color. In the special case when $U=\{u\}$, we simply write $E(u, V), C(u, V)$ and $c(u, V)$ for $E(\{u\}, V), C(\{u\}, V)$ and $c(\{u\}, V)$, respectively. For $v \in V(G)$, let $G-v$ denote the graph obtained from $G$ by deleting the vertex $v$ and the edges incident to $v$. For $U \subseteq V(G)$, let $G-U$ denote the graph obtained from $G$ by deleting all the vertices of $U$ and the edges incident to some vertex of $U$. Moreover, let $G[U]$ denote the subgraph of $G$ induced by $U$, and $C(U)$ denote the set of colors used on the edges of $G[U]$. For any $i \in C(U)$, the subgraph induced by color $i$ means an induced subgraph of $G[U]$ on the edges using color $i$.

\section{Proof of Theorem 1.2}

We begin with the following simple results which will be used later.

Proposition 2.1. If $H_{1}$ is a subgraph of $H_{2}$, then $g r_{k}\left(K_{3}: H_{1}\right) \leq g r_{k}\left(K_{3}: H_{2}\right)$.

Lemma 2.2. ([13]) $r_{2}\left(F_{9}\right)=r_{2}\left(F_{10}\right)=9$.

Lemma 2.3. ([3]) For any integer $k \geq 1$,

$$
\operatorname{gr}_{k}\left(K_{3}: K_{3}\right)= \begin{cases}5^{k / 2}+1, & \text { if } k \text { is even, } \\ 2 \cdot 5^{(k-1) / 2}+1, & \text { if } k \text { is odd. }\end{cases}
$$

Since $F_{9}$ is a subgraph of $F_{10}$, we have $g r_{k}\left(K_{3}: F_{9}\right) \leq g r_{k}\left(K_{3}: F_{10}\right)$ by Proposition 2.1. Thus Theorem 1.2 follows from the following two lemmas immediately.

Lemma 2.4. For any integer $k \geq 1, g_{k}\left(K_{3}: F_{9}\right)> \begin{cases}8 \cdot 5^{(k-2) / 2}, & \text { if } k \text { is even, } \\ 4 \cdot 5^{(k-1) / 2}, & \text { if } k \text { is odd. }\end{cases}$

Proof. If $k$ is even, then let $G_{2}$ be a 2 -colored $K_{8}$ containing no monochromatic $F_{9}$ using colors 1 and 2 . Suppose that $2 i<k$ and we have constructed a $2 i$-coloring $G_{2 i}$ of $K_{n_{2 i}}$ containing neither rainbow $K_{3}$ nor monochromatic $F_{9}$, where $n_{2 i}=8 \cdot 5^{(2 i-2) / 2}$. Let $G^{\prime}$ be a 2-colored $K_{5}$ using colors $2 i+1$ and $2 i+2$ which contains no monochromatic $K_{3}$, i.e., colors $2 i+1$ and $2 i+2$ induce two monochromatic $C_{5}$. We construct $G_{2 i+2}$ by substituting five copies of $G_{2 i}$ into vertices of $G^{\prime}$, i.e., $G_{2 i+2}$ is a blow-up of $G^{\prime}$. Finally, we obtain a $k$-coloring $G_{k}$ of $K_{n}$ containing neither rainbow $K_{3}$ nor monochromatic $F_{9}$, where $n=8 \cdot 5^{(k-2) / 2}$.

If $k$ is odd, then let $G_{1}$ be a monochromatic $K_{4}$ using color 1 . Suppose that $2 i-1<k$ and we have constructed a $(2 i-1)$-coloring $G_{2 i-1}$ of $K_{n_{2 i-1}}$ containing neither rainbow $K_{3}$ nor monochromatic $F_{9}$, where $n_{2 i-1}=4 \cdot 5^{(2 i-2) / 2}$. Let $G^{\prime \prime}$ be a 2-colored $K_{5}$ using colors $2 i$ and $2 i+1$ which contains no monochromatic $K_{3}$. We construct $G_{2 i+1}$ by substituting five copies of $G_{2 i-1}$ into vertices of $G^{\prime \prime}$. Finally, we obtain a $k$-coloring $G_{k}$ of $K_{n}$ containing neither rainbow $K_{3}$ nor monochromatic $F_{9}$, where $n=4 \cdot 5^{(k-1) / 2}$. 
Lemma 2.5. For any integer $k \geq 1, g r_{k}\left(K_{3}: F_{10}\right) \leq \begin{cases}8 \cdot 5^{(k-2) / 2}+1, & \text { if } k \text { is even, } \\ 4 \cdot 5^{(k-1) / 2}+1, & \text { if } k \text { is odd. }\end{cases}$

Proof. We prove the statement by induction on $k$. The case $k=1$ is trivial and the case $k=2$ holds by Lemma 2.2, so we may assume $k \geq 3$. Suppose $G$ is a Gallai- $k$-coloring of $K_{n}$ containing no monochromatic $F_{10}$, where

$$
n= \begin{cases}8 \cdot 5^{(k-2) / 2}+1, & \text { if } k \text { is even, } \\ 4 \cdot 5^{(k-1) / 2}+1, & \text { if } k \text { is odd }\end{cases}
$$

Let $V_{1}, V_{2}, \ldots, V_{m}$ be a Gallai-partition with $\left|V_{1}\right| \geq\left|V_{2}\right| \geq \cdots \geq\left|V_{m}\right|$ and $m \geq 2$. Since $r_{2}\left(F_{10}\right)=9$, we have $2 \leq m \leq 8$. We choose such a partition with $m$ minimum. Suppose colors 1 and 2 are the two colors used between the parts. We first have the following simple facts since $G$ is monochromatic $F_{10}$-free.

Fact 2.6. For any two non-empty parts $V_{i}, V_{j}$ with $c\left(V_{i}, V_{j}\right)=\alpha$, we have

(1) there is no monochromatic $P_{4}$ using color $\alpha$ within $V_{i}$ and $V_{j}$, respectively;

(2) if $\left|V_{i}\right| \geq 3$ and $\left|V_{j}\right| \geq 2$, then $\alpha \notin C\left(V_{i}\right)$;

(3) if $\left|V_{i}\right| \geq 2$ and $\left|V_{j}\right| \geq 2$, then there is no vertex with color $\alpha$ to both $V_{i}$ and $V_{j}$ in $V(G) \backslash\left(V_{i} \cup V_{j}\right)$.

We now claim that $4 \leq m \leq 8$. In fact, if $2 \leq m \leq 3$, then there exists a bipartition $V_{1}$ and $V_{2}$ of $V(G)$ with $\left|C\left(V_{1}, V_{2}\right)\right|=1$, say $c\left(V_{1}, V_{2}\right)=1$. If $\left|V_{2}\right| \geq 2$, then $1 \notin C\left(V_{1}\right)$ by Fact 2.6 (2). Apply induction on $k$ within $V_{1}$, we have $|V(G)|=\left|V_{1}\right|+\left|V_{2}\right| \leq 2\left|V_{1}\right| \leq 2\left[g r_{k-1}\left(K_{3}\right.\right.$ : $\left.\left.F_{10}\right)-1\right]<n$, a contradiction. Thus $\left|V_{2}\right|=1$ and $\left|V_{1}\right|=n-1$. By Fact 2.6 (1), color 1 induces a subgraph $G^{(1)}$ such that each component is a $K_{3}$ or a star within $V_{1}$. In order to avoid a rainbow $K_{3}$ and a monochromatic $P_{4}$ in color 1 , there is only a single color between each pair of components. By Lemma 2.3, we have

$$
g r_{k-1}\left(K_{3}: K_{3}\right)= \begin{cases}2 \cdot 5^{(k-2) / 2}+1, & \text { if } k \text { is even } \\ 5^{(k-1) / 2}+1, & \text { if } k \text { is odd }\end{cases}
$$

Since $\left|V_{1}\right|$ cannot be divided by three and a monochromatic $K_{1,3,3}$ contains a $F_{10}, G^{(1)}$ contains at most $n_{k}=g r_{k-1}\left(K_{3}: K_{3}\right)-2$ components each of which is a $K_{3}$. For $0 \leq i \leq n_{k}$, if there are exactly $n_{k}-i$ components each of which is a $K_{3}$, then there are at most $i+1$ components each of which is a star to avoid a monochromatic $K_{2,2,3}$. Thus after removing at most $2\left(n_{k}-i\right)+i+1$ vertices, there is no edge using color 1 within $V_{1}$. Thus $|V(G)|=\left|V_{1}\right|+\left|V_{2}\right| \leq$ $2\left(n_{k}-i\right)+i+1+\left[g r_{k-1}\left(K_{3}: F_{10}\right)-1\right]+1<n$, a contradiction.

Therefore, we may assume $4 \leq m \leq 8$. Let $r$ be the number of parts with at least three vertices, i.e., $\left|V_{r}\right| \geq 3$ and $\left|V_{r+1}\right| \leq 2$. Since $m \leq 8$ and $|V(G)| \geq 21$, we have $1 \leq r \leq m \leq 8$. We divide the rest of the proof into four cases based on the number $r$.

Case 1. $r=1$.

For $i=1,2$, let $A_{i}$ be the union of parts with color $i$ to $V_{1}$. Since $m \geq 4$, we have $\left|A_{1}\right| \geq 1,\left|A_{2}\right| \geq 1$ and at least one of $A_{1}$ and $A_{2}$ contains more that one vertices, say $\left|A_{1}\right| \geq 2$. Then $1 \notin C\left(V_{1}\right)$ by Fact 2.6 (2), and so $\left|V_{1}\right| \leq g r_{k-1}\left(K_{3}: F_{10}\right)-1$. Since $2 \geq\left|V_{2}\right| \geq \cdots \geq\left|V_{m}\right|$ and $m \leq 8,\left|A_{1}\right|+\left|A_{2}\right| \leq 14$. If $\left|A_{2}\right| \geq 2$, then $2 \notin C\left(V_{1}\right)$ and thus $|V(G)|=\left|V_{1}\right|+\left|A_{1}\right|+\left|A_{2}\right| \leq g r_{k-2}\left(K_{3}: F_{10}\right)-1+14<n$, a contradiction. Thus $\left|A_{2}\right|=1$. Then $\left|A_{1}\right| \geq 3$ since otherwise $|V(G)|=\left|V_{1}\right|+\left|A_{1}\right|+\left|A_{2}\right| \leq g r_{k-1}\left(K_{3}: F_{10}\right)-1+2+1<n$. 
By Fact $2.6(2), 1 \notin C\left(A_{1}\right)$, and thus $\left|A_{1}\right| \leq g r_{k-1}\left(K_{3}: F_{10}\right)-1$. Then $|V(G)|=\left|V_{1}\right|+\left|A_{1}\right|+$ $\left|A_{2}\right| \leq g r_{k-1}\left(K_{3}: F_{10}\right)-1+\min \left\{g r_{k-1}\left(K_{3}: F_{10}\right)-1+1,14\right\}<n$, a contradiction.

Case 2. $r=2$.

In this case, we may assume that $c\left(V_{1}, V_{2}\right)=1$. Then by Fact 2.6 (2), for $i=1,2$, $1 \notin C\left(V_{i}\right)$ and thus $\left|V_{i}\right| \leq g r_{k-1}\left(K_{3}: F_{10}\right)-1$. Since $m \leq 8$ and $2 \geq\left|V_{3}\right| \geq \cdots \geq\left|V_{m}\right|$, if $\left|V_{3} \cup \cdots \cup V_{m}\right| \geq 11$ then there is a monochromatic $K_{1,2,2}$, contradicting to the Fact 2.6 (3). Hence, $\left|V_{3} \cup \cdots \cup V_{m}\right| \leq 10$. By Fact 2.6 (3), we also have that there is no vertex with color 1 to both $V_{1}$ and $V_{2}$. Thus we can partition $V_{3} \cup \cdots \cup V_{m}$ into three parts $A$, $B$ and $C$ such that, $c\left(A, V_{1}\right)=c\left(C, V_{2}\right)=1$ and $c\left(A, V_{2}\right)=c\left(C, V_{1}\right)=c\left(B, V_{1} \cup V_{2}\right)=2$. If $|A \cup B| \geq 2$ and $|C \cup B| \geq 2$, then for $i=1,2$, we have $2 \notin C\left(V_{i}\right)$ and thus $|V(G)|=$ $\left|V_{1}\right|+\left|V_{2}\right|+\left|V_{3} \cup \cdots \cup V_{m}\right| \leq 2\left[g_{k-2}\left(K_{3}: F_{10}\right)-1\right]+10<n$, a contradiction. Thus $|A \cup B| \leq 1$ or $|C \cup B| \leq 1$, say $|C \cup B| \leq 1$, so $|B| \leq 1$.

We first suppose $|B|=1$ and $|C|=0$. Since $m \geq 4,|A| \geq 1$, and thus $2 \notin C\left(V_{2}\right)$. Since $|A|=|V(G)|-\left|V_{1}\right|-\left|V_{2}\right|-|B| \geq n-\left[g r_{k-1}\left(K_{3}: F_{10}\right)-1\right]-\left[g r_{k-2}\left(K_{3}: F_{10}\right)-1\right]-1>2$, we have $1,2 \notin C(A)$. Thus $|V(G)|=\left|V_{1}\right|+\left|V_{2}\right|+|A|+|B|+|C| \leq\left[g r_{k-1}\left(K_{3}: F_{10}\right)-1\right]+2\left[g r_{k-2}\left(K_{3}:\right.\right.$ $\left.\left.F_{10}\right)-1\right]+1<n$, a contradiction. Next we suppose $|B|=0$. Since $m \geq 4$, we have $|A| \geq 1$ and $|C|=1$. If $|A| \geq 2$, then $2 \notin C\left(V_{2}\right)$ and $\left|V_{2}\right| \leq g r_{k-2}\left(K_{3}: F_{10}\right)-1$. Since $|A|=|V(G)|-\left|V_{1}\right|-\left|V_{2}\right|-|C| \geq n-\left[g r_{k-1}\left(K_{3}: F_{10}\right)-1\right]-\left[g r_{k-2}\left(K_{3}: F_{10}\right)-1\right]-1>2$, we have $1,2 \notin C(A)$, and thus $|V(G)| \leq\left[g r_{k-1}\left(K_{3}: F_{10}\right)-1\right]+2\left[g r_{k-2}\left(K_{3}: F_{10}\right)-1\right]+1<n$, a contradiction. Thus, $|A|=1$. By the minimality of $m$, we have $c(A, C)=2$. By Fact 2.6 (1), color 2 induces a subgraph $G^{(2)}$ such that each component is a $K_{3}$ or a star within $V_{1}$. In order to avoid a rainbow $K_{3}$ and a monochromatic $P_{4}$ in color 2 , there is only a single color between each pair of components. Since $1 \notin C\left(V_{1}\right)$ and by Fact 2.6$](3), G^{(2)}$ contains at most $n_{k}^{\prime}=g r_{k-2}\left(K_{3}: K_{3}\right)-1$ components each of which is a $K_{3}$ for avoiding a monochromatic $K_{3,3,3}$ that contains a $F_{10}$. For $0 \leq j \leq n_{k}^{\prime}$, if there are exactly $n_{k}^{\prime}-j$ components each of which is a $K_{3}$, then there are at most $j$ components each of which is a star to avoid a monochromatic $K_{2,3,3}$. Thus after removing at most $2\left(n_{k}^{\prime}-j\right)+j$ vertices, there is no edge using color 2 within $V_{1}$. Thus $|V(G)|=\left|V_{1}\right|+\left|V_{2}\right|+|A|+|C| \leq 2\left|V_{1}\right|+2 \leq$ $2\left[2\left(n_{k}^{\prime}-j\right)+j+\left(g r_{k-2}\left(K_{3}: F_{10}\right)-1\right)\right]+2<n$, a contradiction.

Case 3. $r=3$.

By Fact 2.6 (3), we may assume that $c\left(V_{1}, V_{2} \cup V_{3}\right)=1$ and $c\left(V_{2}, V_{3}\right)=2$. Thus $1 \notin C\left(V_{1}\right) \cup$ $C\left(V_{2}\right) \cup C\left(V_{3}\right)$ and $2 \notin C\left(V_{2}\right) \cup C\left(V_{3}\right)$. By inductive assumption, $\left|V_{1}\right| \leq g r_{k-1}\left(K_{3}: F_{10}\right)-1$, $\left|V_{2}\right| \leq g r_{k-2}\left(K_{3}: F_{10}\right)-1$ and $\left|V_{3}\right| \leq g r_{k-2}\left(K_{3}: F_{10}\right)-1$. Since $m \leq 8$ and $2 \geq\left|V_{4}\right| \geq$ $\cdots \geq\left|V_{m}\right|$, we have $\left|V_{4}\right| \geq \cdots \geq\left|V_{m}\right| \leq 10$. If $c\left(v, V_{1}\right)=1$ for some $v \in V_{4} \cup \cdots \cup V_{m}$, then $c\left(v, V_{2}\right) \neq 1$ and $c\left(v, V_{3}\right) \neq 1$ by Fact 2.6 (3). But then $c\left(v, V_{2}\right)=c\left(v, V_{3}\right)=2$, a contradiction. Thus $c\left(V_{1}, V_{4} \cup \cdots \cup V_{m}\right)=2$. Note that $\left|V_{4} \cup \cdots \cup V_{m}\right|=|V(G)|-\left|V_{1}\right|-\left|V_{2}\right|-\left|V_{3}\right| \geq 5$. By Fact $2.6(2), 2 \notin C\left(V_{1}\right)$ and $2 \notin C\left(V_{4} \cup \cdots \cup V_{m}\right)$. Thus $|V(G)| \leq 3\left[g r_{k-2}\left(K_{3}: F_{10}\right)-1\right]+$ $\min \left\{g r_{k-1}\left(K_{3}: F_{10}\right)-1,10\right\}<n$, a contradiction.

Case 4. $r \geq 4$.

By Fact 2.6 (3), there is no monochromatic $K_{3}$ in the subgraph $H$ of the reduced graph induced on the first four parts. Thus $H$ is one of the two 2-coloring of $K_{4}$ with no monochromatic $K_{3}$. By Fact 2.6 (2), 1,2 $\notin C\left(V_{1}\right) \cup C\left(V_{2}\right) \cup C\left(V_{3}\right) \cup C\left(V_{4}\right)$. Thus for $i \in$ [4], $\left|V_{i}\right| \leq g r_{k-2}\left(K_{3}: F_{10}\right)-1$, and so $\left|V_{5} \cup \cdots \cup V_{m}\right| \geq 5$. If $6 \leq m \leq 8$, then there is a monochromatic $K_{1,2,3}$, a contradiction. Thus $m=5$ and $\left|V_{5}\right| \geq 5$. In order two avoid a monochromatic $F_{10}, G$ is a blow-up of the unique 2-coloring of $K_{5}$ with no monochromatic $K_{3}$. By Fact 2.6 (2), 1,2 $\notin C\left(V_{1}\right) \cup C\left(V_{2}\right) \cup \cdots \cup C\left(V_{5}\right)$. Apply induction on $k$, we have 
$|V(G)| \leq 5\left[g r_{k-2}\left(K_{3}: F_{10}\right)-1\right]<n$, a contradiction.

\section{Ramsey numbers and Gallai-Ramsey numbers for a class of unicyclic graph}

For $n \geq 3$, let $F_{2, n}$ denote the graph obtained by adding $n-2$ pendent edges to a single vertex of $C_{4}$. The vertex with degree $n$ is called a center of $F_{2, n}$. It is easy to see that $\left|V\left(F_{2, n}\right)\right|=\left|E\left(F_{2, n}\right)\right|=n+2$. Note that $F_{2,3}=F_{11}$. In the following, we first give the 2-colored Ramsey number of $F_{2, n}$.

Theorem 3.1. For all integers $k \geq 1$ and $n \geq 3$,

$$
r_{2}\left(F_{2, n}\right)= \begin{cases}2 n-1, & \text { if } n \text { is even, } \\ 2 n, & \text { if } n \text { is odd. }\end{cases}
$$

Proof. Let $n^{\prime}=2 n-\varepsilon$, where $\varepsilon=1$ if $n$ is even, and $\varepsilon=0$ otherwise. Since $K_{1, n}$ is a subgraph of $F_{2, n}$ and $r_{2}\left(K_{1, n}\right)=n^{\prime}$ (proven in [1]), we have $r_{2}\left(F_{2, n}\right) \geq n^{\prime}$.

For the upper bound, suppose $G$ is a 2-coloring of $K_{n^{\prime}}$ containing no monochromatic $F_{2, n}$. Since $r_{2}\left(F_{2,3}\right)=6$ (see [13]), we may assume that $n \geq 4$. Then $G$ contains a monochromatic $K_{1, n}$, say using vertex set $\left\{u, v_{1}, v_{2}, \ldots, v_{n}\right\}$ and $c\left(u,\left\{v_{1}, v_{2}, \ldots, v_{n}\right\}\right)=1$. Let $V(G) \backslash\left\{u, v_{1}, v_{2}, \ldots, v_{n}\right\}=\left\{w_{1}, w_{2}, \ldots, w_{n^{\prime}-n-1}\right\}$. For any $i \in\left[n^{\prime}-n-1\right]$, there are at most one edge using color 1 in $E\left(w_{i},\left\{v_{1}, v_{2}, \ldots, v_{n}\right\}\right)$ in order to avoid a monochromatic $F_{2, n}$. Note that $n \geq 4$ and $n^{\prime}-n-1 \geq 2$. If $c\left(w_{i},\left\{v_{1}, v_{2}, \ldots, v_{n}\right\}\right)=2$ for some $i \in\left[n^{\prime}-n-1\right]$, then it is easy to find a monochromatic $F_{2, n}$ in color 2 , a contradiction. Thus we may consider the following two cases based on $C\left(\left\{w_{1}, w_{2}\right\},\left\{v_{1}, v_{2}, \ldots, v_{n}\right\}\right)$.

Firstly, suppose that $c\left(\left\{w_{1}, w_{2}\right\},\left\{v_{1}, v_{2}, \ldots, v_{n-1}\right\}\right)=2$ and $c\left(\left\{w_{1}, w_{2}\right\}, v_{n}\right)=1$. In order to avoid a monochromatic $F_{2, n}$ in color 2, we have $c\left(\left\{w_{1}, w_{2}\right\}, u\right)=1$. Then $u w_{1} v_{n} w_{2} u$ is a $C_{4}$ in color 1 , which together with $\left\{v_{1}, v_{2}, \ldots, v_{n-2}\right\}$ forms a monochromatic $F_{2, n}$ centered at $u$, a contradiction. Secondly, suppose that $c\left(\left\{w_{1}, w_{2}\right\},\left\{v_{1}, v_{2}, \ldots, v_{n-2}\right\}\right)=c\left(w_{1} v_{n}\right)=$ $c\left(w_{2} v_{n-1}\right)=2$ and $c\left(w_{1} v_{n-1}\right)=c\left(w_{2} v_{n}\right)=1$. Note that we also have $c\left(\left\{w_{1}, w_{2}\right\}, u\right)=1$ similarly as above. If $c\left(v_{1} v_{n-1}\right)=1$, then $u w_{1} v_{n-1} v_{1} u$ is a $C_{4}$ in color 1 , which together with $\left\{v_{2}, v_{3}, \ldots, v_{n-2}, v_{n}\right\}$ forms a monochromatic $F_{2, n}$ centered at $u$, a contradiction. Thus $c\left(v_{1} v_{n-1}\right)=2$, and by symmetry $c\left(\left\{v_{1}, v_{2}, \ldots, v_{n-2}\right\},\left\{v_{n-1}, v_{n}\right\}\right)=2$. If $n=4$, then there is a $F_{2,4}$ in color 2 centered at $v_{1}$. If $n \geq 5$, then $n^{\prime}-n-1 \geq 4$ and we may assume $c\left(w_{3} v_{n-1}\right)=2$ without loss of generality. Then $v_{n-1} v_{1} v_{n} v_{2} v_{n-1}$ is a $C_{4}$ in color 2 , which together with $\left\{v_{3}, v_{4}, \ldots, v_{n-2}, w_{2}, w_{3}\right\}$ forms a monochromatic $F_{2, n}$ centered at $v_{n-1}$, a contradiction.

\section{Theorem 3.2.}

(1) For all integers $k \geq 1$ and $n \in\{3,4\}, g r_{k}\left(K_{3}: F_{2, n}\right)=r_{2}\left(F_{2, n}\right)+k-2$;

(2) For any integer $k \geq 3, g r_{k}\left(K_{3}: F_{2,5}\right)=k+9$;

(3) For all integers $k \geq 3$ and $n \geq 6$,

$$
k(n-1)+2 \geq g r_{k}\left(K_{3}: F_{2, n}\right) \geq \begin{cases}\frac{5 n}{2}+k-6, & \text { if } n \text { is even, } \\ \frac{5 n-1}{2}+k-4, & \text { if } n \text { is odd. }\end{cases}
$$

Proof. We first prove the lower bounds by constructing some appropriate Gallai- $k$-colorings of complete graphs without monochromatic $F_{2, n}$. For $n \in\{3,4\}$, let $G_{2}$ be a 2-coloring of 
complete graph on $r_{2}\left(F_{2, n}\right)-1$ vertices using colors 1 and 2 without monochromatic $F_{2, n}$. Let $G^{\prime}$ be the unique 2-coloring of $K_{5}$ using colors 2 and 3 with no monochromatic $K_{3}$. For $n \geq 5$ and $n$ even, let $G_{3}$ be the 3 -coloring obtained by substituting $K_{n / 2}$ (using only color 1 ) into one vertex of $G^{\prime}$ and $K_{n / 2-1}$ (using only color 1 ) into the other four vertices of $G^{\prime}$. For $n \geq 5$ and $n$ odd, let $G_{3}$ be the 3 -coloring obtained by substituting $K_{(n-1) / 2}$ (using only color 1 ) into five vertex of $G^{\prime}$. Suppose $i<k$ and we have constructed $G_{i}$ containing no rainbow $K_{3}$ and no monochromatic $F_{2, n}$. We construct $G_{i+1}$ by adding a new vertex to $G_{i}$ such that all the new edges are colored by color $i+1$. Finally, we obtain a $k$-coloring $G_{k}$ containing no rainbow $K_{3}$ and no monochromatic $F_{2, n}$. For $n \in\{3,4\},\left|V\left(G_{k}\right)\right|=r_{2}\left(F_{2, n}\right)+k-3$. For $n \geq 6$, we have

$$
\left|V\left(G_{k}\right)\right|= \begin{cases}\frac{5 n}{2}+k-7, & \text { if } n \text { is even, } \\ \frac{5 n-1}{2}+k-5, & \text { if } n \text { is odd. }\end{cases}
$$

For $n=5$, we construct $G_{k+1}$ by adding a new vertex to $G_{k}$ such that all the new edges are colored by color 1 . Then $G_{k+1}$ contains no rainbow $K_{3}$ and no monochromatic $F_{2,5}$, and $\left|V\left(G_{k}\right)\right|=k+8$.

For the upper bound, let

$$
n_{k}^{\prime}= \begin{cases}r_{2}\left(F_{2, n}\right)+k-2, & \text { if } n \in\{3,4\}, \\ k+9, & \text { if } n=5, \\ k(n-1)+2, & \text { if } n \geq 6 .\end{cases}
$$

We will prove that every Gallai- $k$-coloring of $K_{n_{k}^{\prime}}$ contains a monochromatic $F_{2, n}$ for all integers $k \geq 1$ and $n \geq 3$. The case $k=1$ is trivial and the case $k=2$ holds by Theorem 3.1 , so we may assume $k \geq 3$ in the following. For a contradiction, suppose $G$ is a $k$-coloring of $K_{n^{\prime}}$ containing no rainbow $K_{3}$ and no monochromatic $F_{2, n}$. We choose such a $G$ with $k$ minimal. Let $V_{1}, V_{2}, \ldots, V_{m}$ be a Gallai-partition with $\left|V_{1}\right| \geq\left|V_{2}\right| \geq \cdots \geq\left|V_{m}\right|$ and $2 \leq m \leq r_{2}\left(F_{2, n}\right)-1$. Suppose colors 1 and 2 are the two colors used between the parts.

We first suppose that $m \geq 4$. In this case, we have $\left|V(G) \backslash V_{1}\right| \geq 3$. In order to avoid a monochromatic $F_{2, n}$ and since $k \geq 3$, we have $2 \leq\left|V_{1}\right| \leq n-1$. For $i=1,2$, let $A_{i}$ be the union of parts with color $i$ to $V_{1}$. If $n \notin\{4,5\}$, then $\max \left\{\left|A_{1}\right|,\left|A_{2}\right|\right\} \geq\left\lceil\frac{|V(G)|-\left|V_{1}\right|}{2}\right\rceil \geq\left\lceil\frac{n_{k}^{\prime}-(n-1)}{2}\right\rceil \geq$ $n$, which implies that there is a monochromatic $F_{2, n}$, a contradiction. If $n=5$, we must have $\left|V_{1}\right|=\left|A_{1}\right|=\left|A_{2}\right|=4$ and $k=3$ by a similar argument. For any edge $u v \in E\left(A_{1}, A_{2}\right)$, we have $c(u v) \in\{1,2\}$, resulting in a monochromatic $F_{2,5}$, a contradiction. If $n=4$, we have $2 \leq\left|V_{1}\right| \leq 3$ and $3 \geq \max \left\{\left|A_{1}\right|,\left|A_{2}\right|\right\} \geq\left\lceil\frac{k+5-3}{2}\right\rceil \geq 3$, say $\left|A_{1}\right|=3$. If $\left|V_{1}\right|=2$, then $\left|A_{2}\right|=3$ and $k=3$. Let $A_{1}=\left\{u_{1}, u_{2}, u_{3}\right\}$ and $A_{2}=\left\{v_{1}, v_{2}, v_{3}\right\}$. In order to avoid a monochromatic $F_{2,4}$, there are at most one edge using color 1 between $u_{i}$ and $A_{2}$ for each $i \in$ [3]. Thus there are at most three edges using color 1 between $A_{1}$ and $A_{2}$. Then there are at least six edges using color 2 between $A_{1}$ and $A_{2}$, and in particular, there exist a vertex $v_{i} \in A_{2}$ such that there are at least two edges using color 2 between $v_{i}$ and $A_{1}$, resulting in a monochromatic $F_{2,4}$, a contradiction. If $\left|V_{1}\right|=3$, then $2 \leq\left|A_{2}\right| \leq 3$. For avoiding a monochromatic $F_{2,4}$ in color $1,1 \notin C\left(A_{1}, A_{2}\right)$, i.e., $c\left(A_{1}, A_{2}\right)=2$, resulting in a monochromatic $F_{2,4}$ in color 2 , a contradiction.

Therefore, we have $2 \leq m \leq 3$. In this case, there exists a bipartition $V_{1}, V_{2}$ of $V(G)$ with $\left|C\left(V_{1}, V_{2}\right)\right|=1$, say $c\left(V_{1}, V_{2}\right)=1$. Since $k \geq 3,\left|V_{1}\right| \geq\left\lceil\frac{|V(G)|}{2}\right\rceil \geq n$. Thus $\left|V_{2}\right|=1$ for avoiding a monochromatic $F_{2, n}$ in color 1 . Moreover, if $1 \notin C\left(V_{1}\right)$, then $|V(G)|=\left|V_{1}\right|+\left|V_{2}\right| \leq$ 
$\left(n_{k-1}^{\prime}-1\right)+1<n_{k}^{\prime}$ by the minimality of $k$, a contradiction. Thus $1 \in C\left(V_{1}\right)$, say $v_{1}, v_{2} \in V_{1}$ and $c\left(v_{1} v_{2}\right)=1$. In order to avoid a monochromatic $F_{2, n}$ in color $1,1 \notin C\left(\left\{v_{1}, v_{2}\right\}, V_{1} \backslash\left\{v_{1}, v_{2}\right\}\right)$. Since $G$ is rainbow $K_{3}$-free, we have $c\left(v_{1} v\right)=c\left(v_{2} v\right)$ for any $v \in V_{1} \backslash\left\{v_{1}, v_{2}\right\}$. For avoiding a monochromatic $F_{2, n}$, there are at most $n-1$ vertices in $V_{1} \backslash\left\{v_{1}, v_{2}\right\}$ with a single color to $\left\{v_{1}, v_{2}\right\}$. Note that $\left|V_{1} \backslash\left\{v_{1}, v_{2}\right\}\right|=n_{k}^{\prime}-3$ and $\left|C\left(\left\{v_{1}, v_{2}\right\}, V_{1} \backslash\left\{v_{1}, v_{2}\right\}\right)\right|=k-1$.

If $n \geq 6$, then there are at least $\left\lceil\frac{n_{k}^{\prime}-3}{k-1}\right\rceil \geq n$ vertices in $V_{1} \backslash\left\{v_{1}, v_{2}\right\}$ with a single color to $\left\{v_{1}, v_{2}\right\}$, i.e., there is a monochromatic $F_{2, n}$, a contradiction.

If $n=3$, then there exist four vertices $u_{1}, u_{2}, u_{3}, u_{4} \in V_{1} \backslash\left\{v_{1}, v_{2}\right\}$ such that $c\left(\left\{v_{1}, v_{2}\right\}\right.$, $\left.\left\{u_{1}, u_{2}\right\}\right)=i$ and $c\left(\left\{v_{1}, v_{2}\right\},\left\{u_{3}, u_{4}\right\}\right)=j$, where $2 \leq i<j \leq k$. Since $G$ is rainbow $K_{3}$-free, we have $C\left(\left\{u_{1}, u_{2}\right\},\left\{u_{3}, u_{4}\right\}\right) \subseteq\{i, j\}$. Then there is a monochromatic $F_{2,3}$ in color $i$ or $j$, a contradiction.

If $n=4$, then we may consider the following two cases. First, there exist five vertices $u_{1}, u_{2}, \ldots, u_{5} \in V_{1} \backslash\left\{v_{1}, v_{2}\right\}$ such that $c\left(\left\{v_{1}, v_{2}\right\},\left\{u_{1}, u_{2}, u_{3}\right\}\right)=i$ and $c\left(\left\{v_{1}, v_{2}\right\},\left\{u_{4}, u_{5}\right\}\right)=j$, where $2 \leq i<j \leq k$. Since $G$ is rainbow $K_{3}$-free, we have $C\left(\left\{u_{1}, u_{2}, u_{3}\right\},\left\{u_{4}, u_{5}\right\}\right) \subseteq\{i, j\}$. In order to avoid a monochromatic $F_{2,4}$ in color $j$, there are at least two edges using color $i$ between $u_{4}$ (resp., $u_{5}$ ) and $\left\{u_{1}, u_{2}, u_{3}\right\}$. Thus there exists a vertex, say $u_{1}$, such that $c\left(u_{1},\left\{u_{4}, u_{5}\right\}\right)=i$, resulting in a monochromatic $F_{2,4}$, a contradiction. Second, there exist six vertices $u_{1}, u_{2}, \ldots, u_{6} \in V_{1} \backslash\left\{v_{1}, v_{2}\right\}$ such that $c\left(\left\{v_{1}, v_{2}\right\},\left\{u_{1}, u_{2}\right\}\right)=i, c\left(\left\{v_{1}, v_{2}\right\},\left\{u_{3}, u_{4}\right\}\right)=$ $j$ and $c\left(\left\{v_{1}, v_{2}\right\},\left\{u_{5}, u_{6}\right\}\right)=l$, where $2 \leq i<j<l \leq k$. Without loss of generality,we may assume that $c\left(u_{1} u_{3}\right)=i$. Then $c\left(u_{1} u_{4}\right)=j$ and $c\left(u_{1},\left\{u_{5}, u_{6}\right\}\right)=l$. Since $c\left(u_{4} u_{5}\right) \in\{j, l\}$, there is a monochromatic $F_{2,4}$ in color $j$ or $l$, a contradiction.

If $n=5$, then by the pigeonhole principle, we may consider the following two cases. First, there exist nine vertices $u_{1}, u_{2}, \ldots, u_{9} \in V_{1} \backslash\left\{v_{1}, v_{2}\right\}$ such that $c\left(\left\{v_{1}, v_{2}\right\},\left\{u_{1}, u_{2}, u_{3}, u_{4}\right\}\right)=i$, $c\left(\left\{v_{1}, v_{2}\right\},\left\{u_{5}, u_{6}, u_{7}\right\}\right)=j$ and $c\left(\left\{v_{1}, v_{2}\right\},\left\{u_{8}, u_{9}\right\}\right)=l$, where $2 \leq i<j<l \leq k$. In order to avoid a monochromatic $F_{2,5}$ in color $j$ (resp., $l$ ), there are at most two edges using color $j$ (resp., $l$ ) between each $u \in\left\{u_{5}, u_{6}, u_{7}\right\}$ (resp., $\left\{u_{8}, u_{9}\right\}$ ) and $\left\{u_{1}, u_{2}, u_{3}, u_{4}\right\}$. Thus, there are at least ten edges using color $i$ between $\left\{u_{1}, u_{2}, u_{3}, u_{4}\right\}$ and $\left\{u_{5}, u_{6}, \ldots, u_{9}\right\}$, and in particular, there exist a vertex $u \in\left\{u_{1}, u_{2}, u_{3}, u_{4}\right\}$ such that there are at least three edges using color $i$ between $u$ and $\left\{u_{5}, u_{6}, \ldots, u_{9}\right\}$, resulting in a monochromatic $F_{2,5}$, a contradiction. Second, there exist eight vertices $u_{1}, u_{2}, \ldots, u_{8} \in V_{1} \backslash\left\{v_{1}, v_{2}\right\}$ such that $c\left(\left\{v_{1}, v_{2}\right\},\left\{u_{1}, u_{2}\right\}\right)=i$, $c\left(\left\{v_{1}, v_{2}\right\},\left\{u_{3}, u_{4}\right\}\right)=j, c\left(\left\{v_{1}, v_{2}\right\},\left\{u_{5}, u_{6}\right\}\right)=s$ and $c\left(\left\{v_{1}, v_{2}\right\},\left\{u_{7}, u_{8}\right\}\right)=t$, where $2 \leq i<$ $j<s<t \leq k$. Since there are at most two edges using color $i$ in $E\left(u_{1},\left\{u_{3}, u_{4}, \ldots, u_{8}\right\}\right)$, we may assume that $c\left(u_{1},\left\{u_{7}, u_{8}\right\}\right)=t$ without loss of generality. Then there is at most one edge using color $t$ in $E\left(u_{7},\left\{u_{3}, u_{4}, u_{5}, u_{6}\right\}\right)$, we may assume that $c\left(u_{7},\left\{u_{5}, u_{6}\right\}\right)=s$. For avoiding a rainbow $K_{3}$, we have $c\left(u_{1},\left\{u_{5}, u_{6}\right\}\right)=s$. For avoiding a monochromatic $F_{2,5}$ in color $s$, we have $c\left(u_{8},\left\{u_{5}, u_{6}\right\}\right)=t$, resulting in a monochromatic $F_{2,5}$ in color $t$ with center $u_{8}$, a contradiction.

\section{Proof of Theorem 1.4}

We begin with the following Ramsey numbers which will be used in the proofs.

Lemma 4.1. ([13] $) r_{2}\left(F_{12}\right)=r_{2}\left(F_{13}\right)=10$.

Theorem 1.4 follows from the following two lemmas immediately. 
Lemma 4.2. For any integer $k \geq 1, H \in\left\{F_{12}, F_{13}\right\}$,

$$
g r_{k}\left(K_{3}: H\right)> \begin{cases}9 \cdot 5^{(k-2) / 2}, & \text { if } k \text { is even } \\ 4 \cdot 5^{(k-1) / 2}, & \text { if } k \text { is odd } .\end{cases}
$$

Proof. If $k$ is even, then let $G_{2}$ be a 2-colored $K_{9}$ containing no monochromatic $H$ using colors 1 and 2. Suppose that $2 i<k$ and we have constructed a $2 i$-coloring $G_{2 i}$ of $K_{n_{2 i}}$ containing neither rainbow $K_{3}$ nor monochromatic $H$, where $n_{2 i}=9 \cdot 5^{(2 i-2) / 2}$. Let $G^{\prime}$ be a 2-colored $K_{5}$ using colors $2 i+1$ and $2 i+2$ which contains no monochromatic $K_{3}$, i.e., colors $2 i+1$ and $2 i+2$ induce two monochromatic $C_{5}$. We construct $G_{2 i+2}$ by substituting five copies of $G_{2 i}$ into vertices of $G^{\prime}$, i.e., $G_{2 i+2}$ is a blow-up of $G^{\prime}$. Finally, we obtain a $k$-coloring $G_{k}$ of $K_{n}$ containing neither rainbow $K_{3}$ nor monochromatic $H$, where $n=9 \cdot 5^{(k-2) / 2}$.

If $k$ is odd, then let $G_{1}$ be a monochromatic $K_{4}$ using color 1 . Suppose that $2 i-1<k$ and we have constructed a $(2 i-1)$-coloring $G_{2 i-1}$ of $K_{n_{2 i-1}}$ containing neither rainbow $K_{3}$ nor monochromatic $H$, where $n_{2 i-1}=4 \cdot 5^{(2 i-2) / 2}$. Let $G^{\prime \prime}$ be a 2-colored $K_{5}$ using colors $2 i$ and $2 i+1$ which contains no monochromatic $K_{3}$. We construct $G_{2 i+1}$ by substituting five copies of $G_{2 i-1}$ into vertices of $G^{\prime \prime}$. Finally, we obtain a $k$-coloring $G_{k}$ of $K_{n}$ containing neither rainbow $K_{3}$ nor monochromatic $H$, where $n=4 \cdot 5^{(k-1) / 2}$.

Lemma 4.3. For any integer $k \geq 1, H \in\left\{F_{12}, F_{13}\right\}$,

$$
\operatorname{gr}_{k}\left(K_{3}: H\right) \leq \begin{cases}9 \cdot 5^{(k-2) / 2}+1, & \text { if } k \text { is even }, \\ 4 \cdot 5^{(k-1) / 2}+1, & \text { if } k \text { is odd. }\end{cases}
$$

Proof. We prove the statement by induction on $k$. The case $k=1$ is trivial and the case $k=2$ holds by Lemma 4.1, so we may assume $k \geq 3$. Suppose $G$ is a Gallai- $k$-coloring of $K_{n}$ containing no monochromatic $H$, where

$$
n= \begin{cases}9 \cdot 5^{(k-2) / 2}+1, & \text { if } k \text { is even, } \\ 4 \cdot 5^{(k-1) / 2}+1, & \text { if } k \text { is odd. }\end{cases}
$$

Let $V_{1}, V_{2}, \ldots, V_{m}$ be a Gallai-partition with $\left|V_{1}\right| \geq\left|V_{2}\right| \geq \cdots \geq\left|V_{m}\right|$ and $2 \leq m \leq 9$. We choose such a partition with $m$ minimum. Suppose colors 1 and 2 are the two colors used between the parts. We first have the following simple facts since $G$ is monochromatic $H$-free.

Fact 4.4. For any two non-empty parts $V_{i}, V_{j}$ with $c\left(V_{i}, V_{j}\right)=\alpha$, we have

(1) there is no monochromatic $P_{4}$ using color $\alpha$ within $V_{i}$ and $V_{j}$, respectively;

(2) if $\left|V_{i}\right| \geq 3$ and $\left|V_{j}\right| \geq 3$, then $\alpha \notin C\left(V_{i}\right)$ and $\alpha \notin C\left(V_{i}\right)$, respectively;

(3) if $\left|V_{i}\right| \geq 3$ and $\left|V_{j}\right| \geq 2$, then there is no monochromatic $P_{3}$ using color $\alpha$ within $V_{i}$;

(4) if $\left|V_{i}\right| \geq 4$, then there is no monochromatic $K_{3}$ using color $\alpha$ within $V_{i}$;

(5) if $\left|V_{i}\right| \geq 2$ and $\left|V_{j}\right| \geq 2$, then there is no vertex with color $\alpha$ to both $V_{i}$ and $V_{j}$ in $V(G) \backslash\left(V_{i} \cup V_{j}\right)$.

Fact 4.5. For any two non-empty parts $V_{i}, V_{j}$ with $c\left(V_{i}, V_{j}\right)=\alpha$, we have

(1) if $H=F_{12}$ and $\left|V_{i}\right| \geq 4$, then there is no monochromatic $P_{3}$ using color $\alpha$ within $V_{i}$;

(2) if $H=F_{13},\left|V_{i}\right| \geq 3$ and $\left|V_{j}\right| \geq 2$, then $\alpha \notin C\left(V_{i}\right)$. 
We now claim that $4 \leq m \leq 9$. In fact, if $2 \leq m \leq 3$, then there exists a bipartition $V_{1}$ and $V_{2}$ of $V(G)$ with $\left|C\left(V_{1}, V_{2}\right)\right|=1$, say $c\left(V_{1}, V_{2}\right)=1$. If $\left|V_{2}\right| \geq 3$, then $1 \notin C\left(V_{1}\right)$ by Fact 4.4 (2). Apply induction on $k$ within $V_{1}$, we have $|V(G)|=\left|V_{1}\right|+\left|V_{2}\right| \leq 2\left|V_{1}\right| \leq 2\left[g_{k-1}\left(K_{3}\right.\right.$ : $H)-1]<n$, a contradiction. If $\left|V_{2}\right|=2$, then by Fact 4.4 (3) color 1 induces a matching within $V_{1}$. If $\left|V_{2}\right|=1$, then by Fact 4.4 (1) and (4), color 1 induces a subgraph such that each component is a star within $V_{1}$. Thus when $1 \leq\left|V_{2}\right| \leq 2$, we can partition $V_{1}$ into two subsets $V_{1}^{\prime}$ and $V_{1}^{\prime \prime}$ such that there is no color 1 within $V_{1}^{\prime}$ and $V_{1}^{\prime \prime}$, respectively. By inductive assumption, $|V(G)|=\left|V_{1}^{\prime}\right|+\left|V_{1}^{\prime \prime}\right|+\left|V_{2}\right| \leq 2\left[g r_{k-1}\left(K_{3}: H\right)-1\right]+2<n$, a contradiction.

Therefore, we may assume $4 \leq m \leq 9$. Let $r$ be the number of parts with at least three vertices, i.e., $\left|V_{r}\right| \geq 3$ and $\left|V_{r+1}\right| \leq 2$. Since $m \leq 9$ and $|V(G)| \geq 21$, we have $1 \leq r \leq m \leq 9$. We divide the rest of the proof into four cases based on the number $r$.

Case 1. $r \geq 4$.

By Fact 4.4 (5), there is no monochromatic $K_{3}$ in the subgraph $R$ of the reduced graph induced on the first four parts. Thus $R$ is one of the two 2-coloring of $K_{4}$ with no monochromatic $K_{3}$. If $m=5$, then the reduced graph is the unique 2-coloring of $K_{5}$ with no monochromatic $K_{3}$. Thus for $4 \leq m \leq 5$, we have $1,2 \notin C\left(V_{1}\right)$ by Fact 4.4 (2), and so $|V(G)| \leq 5\left|V_{1}\right| \leq 5\left[g_{k-2}\left(K_{3}: H\right)-1\right]<n$, a contradiction. If $6 \leq m \leq 9$, then $1 \geq\left|V_{5}\right| \geq \cdots \geq\left|V_{m}\right|$ otherwise there is a monochromatic $K_{1,2,3}$. Since $n=|V(G)|=$ $\sum_{i=1}^{4}\left|V_{i}\right|+\sum_{j=5}^{m}\left|V_{j}\right| \leq 4\left|V_{1}\right|+5 \leq 4\left[g r_{k-2}\left(K_{3}: H\right)-1\right]+5$, we have $n=21, k=3,\left|V_{i}\right|=4$ for $i \in[4]$ and $\left|V_{j}\right|=1$ for $j \in\{5,6, \ldots, 9\}$. Note that there is a monochromatic $K_{3}$ in subgraph of the reduced graph induced on $V_{1}, V_{2}, \ldots, V_{6}$, i.e., there is a monochromatic $K_{1,3,3}$ or $K_{1,1,3}$ in $G$. If the former holds, then we are done. If the latter holds, then there is a monochromatic $F_{12}$ using six edges of the $K_{1,3,3}$, and a monochromatic $F_{13}$ using five edges of the $K_{1,3,3}$ and one edge between the first four parts, a contradiction.

Case 2. $r=3$.

In order to avoid a monochromatic $K_{3,3,3}$, we may assume that $c\left(V_{1}, V_{2} \cup V_{3}\right)=1$ and $c\left(V_{2}, V_{3}\right)=2$. Then $1 \notin C\left(V_{1}\right) \cup C\left(V_{2}\right) \cup C\left(V_{3}\right)$ and $2 \notin C\left(V_{2}\right) \cup C\left(V_{3}\right)$. By inductive assumption, $\left|V_{1}\right| \leq g r_{k-1}\left(K_{3}: H\right)-1,\left|V_{2}\right| \leq g r_{k-2}\left(K_{3}: H\right)-1$ and $\left|V_{3}\right| \leq g r_{k-2}\left(K_{3}: H\right)-1$, and so $\left|V_{4} \cup \cdots \cup V_{m}\right| \geq 4$. Note that $\left|V_{4} \cup \cdots \cup V_{m}\right| \leq 10$, otherwise there is a monochromatic $K_{1,2,2}$ since $m \leq 9$ and $2 \geq\left|V_{4}\right| \geq \cdots \geq\left|V_{m}\right|$. If $c\left(v, V_{1}\right)=1$ for some $v \in V_{4} \cup \cdots \cup V_{m}$, then $c\left(v, V_{2}\right) \neq 1$ and $c\left(v, V_{3}\right) \neq 1$ by Fact $4.4(5)$. But then $c\left(v, V_{2}\right)=c\left(v, V_{3}\right)=2$, a contradiction. Thus $c\left(V_{1}, V_{4} \cup \cdots \cup V_{m}\right)=2$. Recall that we have $\left|V_{4} \cup \cdots \cup V_{m}\right| \geq 4$, and thus $2 \notin C\left(V_{1}\right)$ and $2 \notin C\left(V_{4} \cup \cdots \cup V_{m}\right)$ by Fact $4.4(2)$. Hence, $\left|V_{1}\right| \leq g r_{k-2}\left(K_{3}: H\right)-1$ and $\left|V_{4} \cup \cdots \cup V_{m}\right| \leq \min \left\{10, g r_{k-1}\left(K_{3}: H\right)-1\right\}$. Since $n=|V(G)|=\sum_{i=1}^{3}\left|V_{i}\right|+\sum_{j=4}^{m}\left|V_{j}\right| \leq$ $3\left[g r_{k-2}\left(K_{3}: H\right)-1\right]+\min \left\{10, g r_{k-1}\left(K_{3}: H\right)-1\right\}$, we have $n=21, k=3$ and $\left|V_{4} \cup \cdots \cup V_{m}\right|=$ 9. For any vertex $v \in V_{4} \cup \cdots \cup V_{m}$, at least one of $c\left(v, V_{2}\right)$ and $c\left(v, V_{3}\right)$ is 1 . Thus there are at least five vertices in $V_{4} \cup \cdots \cup V_{m}$, say $\left\{x_{1}, x_{2}, \ldots, x_{5}\right\}$, such that $c\left(\left\{x_{1}, x_{2}, \ldots, x_{5}\right\}, V_{2}\right)=1$ or $c\left(\left\{x_{1}, x_{2}, \ldots, x_{5}\right\}, V_{3}\right)=1$. Then $1 \notin\left\{x_{1}, x_{2}, \ldots, x_{5}\right\}$, a contradiction.

Case 3. $r=1$.

For $i=1,2$, let $A_{i}$ be the union of parts with color $i$ to $V_{1}$. Then $\left|A_{1}\right|+\left|A_{2}\right| \leq 16$. If $\left|A_{1}\right| \geq 3$ and $\left|A_{2}\right| \geq 3$, then $1,2 \notin C\left(V_{1}\right)$ by Fact 4.4 (2). By inductive assumption, $|V(G)|=\left|V_{1}\right|+\left|A_{1}\right|+\left|A_{2}\right| \leq g r_{k-2}\left(K_{3}: H\right)-1+16<n$, a contradiction. If $\left|A_{1}\right| \geq 3$ and $\left|A_{2}\right| \leq 2$, then $1 \notin C\left(V_{1}\right)$ and $1 \notin C\left(A_{1}\right)$ by Fact 4.4 (2). By inductive assumption, $|V(G)|=\left|V_{1}\right|+\left|A_{1}\right|+\left|A_{2}\right| \leq 2\left[g r_{k-1}\left(K_{3}: H\right)-1\right]+2<n$, a contradiction. Thus $\left|A_{1}\right| \leq 2$, and by symmetry $\left|A_{2}\right| \leq 2$. Since $m \geq 4$, we have $\left|A_{1}\right| \geq 1,\left|A_{2}\right| \geq 1$ and $\max \left\{\left|A_{1}\right|,\left|A_{2}\right|\right\}=2$, say $\left|A_{1}\right|=2$. 
If $H=F_{12}$, then there is no monochromatic $P_{3}$ using color 1 (resp., color 2) within $V_{1}$ by Fact 4.5 (1). Thus there is no rainbow $P_{3}$ using colors 1 and 2, since otherwise there is a monochromatic $P_{3}$ using color 1 or 2 since $G$ is rainbow $K_{3}$-free. Hence, colors 1 and 2 induce a matching within $V_{1}$. Then we can remove at most $\left\lfloor\frac{\left|V_{1}\right|}{2}\right\rfloor$ vertices such that there is no color 1 and no color 2 within $V_{1}$. By inductive assumption, $|V(G)|=\left|V_{1}\right|+\left|A_{1}\right|+\left|A_{2}\right| \leq$ $2\left[\operatorname{gr}_{k-2}\left(K_{3}: F_{12}\right)-1\right]+4<n$, a contradiction.

If $H=F_{13}$, then $1 \notin C\left(V_{1}\right)$ by Fact $4.5(2)$. Now $|V(G)|=\left|V_{1}\right|+\left|A_{1}\right|+\left|A_{2}\right| \leq g r_{k-1}\left(K_{3}\right.$ : $\left.F_{13}\right)-1+4<n$, a contradiction.

Case 4. $r=2$.

In this case, we may assume that $c\left(V_{1}, V_{2}\right)=1$. Then by Fact 4.4 (2), for $i=1,2$, $1 \notin C\left(V_{i}\right)$ and thus $\left|V_{i}\right| \leq g r_{k-1}\left(K_{3}: H\right)-1$. Since $m \leq 9$ and $2 \geq\left|V_{3}\right| \geq \cdots \geq\left|V_{m}\right|$, if $\left|V_{3} \cup \cdots \cup V_{m}\right| \geq 12$ then there is a monochromatic $K_{1,2,2}$, contradicting to the Fact 4.4 (5). Hence, $\left|V_{3} \cup \cdots \cup V_{m}\right| \leq 11$. By Fact 4.4 (5), we also have that there is no vertex with color 1 to both $V_{1}$ and $V_{2}$. Thus we can partition $V_{3} \cup \cdots \cup V_{m}$ into three parts $A, B$ and $C$ such that, $c\left(A, V_{1}\right)=c\left(C, V_{2}\right)=1$ and $c\left(A, V_{2}\right)=c\left(C, V_{1}\right)=c\left(B, V_{1} \cup V_{2}\right)=2$. Note that we have $|A \cup B| \leq 2$ or $|B \cup C| \leq 2$, otherwise $2 \notin C\left(V_{1}\right)$ and $2 \notin C\left(V_{2}\right)$ by Fact 4.4 (2), and then $|V(G)|=\left|V_{1}\right|+\left|V_{2}\right|+|A|+|B|+|C| \leq 2\left[g_{k-2}\left(K_{3}: H\right)-1\right]+11<n$, a contradiction. Without loss of generality, let $|B \cup C| \leq 2$, so $|B| \leq 2,|C| \leq 2$.

If $H=F_{12}$, then we first suppose $|B|=2$ and $|C|=0$. Since $m \geq 4,|A| \neq 0$. If $|A| \geq 3$, then $2 \notin C\left(V_{2}\right)$ and $1,2 \notin C(A)$. Thus $|V(G)|=\left|V_{1}\right|+\left|V_{2}\right|+|A|+|B|+|C| \leq\left[g r_{k-1}\left(K_{3}\right.\right.$ : $\left.\left.F_{12}\right)-1\right]+2\left[g_{k-2}\left(K_{3}: F_{12}\right)-1\right]+2<n$, a contradiction. Hence, $|A| \leq 2$, but then $|V(G)|=\left|V_{1}\right|+\left|V_{2}\right|+|A|+|B|+|C| \leq\left[g r_{k-1}\left(K_{3}: F_{12}\right)-1\right]+\left[g r_{k-2}\left(K_{3}: F_{12}\right)-1\right]+4<n$, a contradiction. Next, we suppose $|B|=1$ and $|C| \leq 1$. If $|A| \geq 2$, then we can derive a contradiction similarly as above. Thus $|A| \leq 1$. If $|A|=0$ or $|C|=0$, then $|V(G)| \leq$ $2\left[\operatorname{gr}_{k-1}\left(K_{3}: F_{12}\right)-1\right]+2<n$. Hence, $|A|=|C|=1$. By Fact 4.4 (3), color 2 induces a matching within $V_{1}$ and $V_{2}$. For $i=1,2$, there is no color 2 within $V_{i}$ after removing at most $\left\lfloor\frac{\left|V_{i}\right|}{2}\right\rfloor$ vertices. Thus $|V(G)| \leq 4\left[g_{k-2}\left(K_{3}: F_{12}\right)-1\right]+4<n$, a contradiction. Finally, we suppose $|B|=0$ and $|C| \leq 2$. If $|A| \geq 3$, then $2 \notin C\left(V_{2}\right)$ and $1,2 \notin C(A)$ but then $|V(G)| \leq\left[g r_{k-1}\left(K_{3}: F_{12}\right)-1\right]+2\left[g r_{k-2}\left(K_{3}: F_{12}\right)-1\right]+2<n$. Thus $|A| \leq 2$. Note $\left|V_{2}\right| \geq 4$ otherwise $|V(G)| \leq\left[g r_{k-1}\left(K_{3}: F_{12}\right)-1\right]+3+4<n$. Since $m \geq 4$, we have $|A| \neq 0$ and $|C| \neq 0$. By Fact 4.5 (1), color 2 induces a matching within $V_{1}$ and $V_{2}$. Thus $|V(G)| \leq 4\left[g r_{k-2}\left(K_{3}: F_{12}\right)-1\right]+4<n$, a contradiction.

If $H=F_{13}$, then $|A \cup B| \leq 1$ or $|B \cup C| \leq 1$, otherwise $2 \notin C\left(V_{1}\right)$ and $2 \notin C\left(V_{2}\right)$ by Fact 4.5 (2), and then $|V(G)|=\left|V_{1}\right|+\left|V_{2}\right|+|A|+|B|+|C| \leq 2\left[g_{k-2}\left(K_{3}: F_{13}\right)-1\right]+11<n$, a contradiction. Without loss of generality, let $|B \cup C| \leq 1$, so $|B| \leq 1,|C| \leq 1$. We first suppose $|B|=1$ and $|C|=0$. Since $m \geq 4,|A| \neq 0$. Then $2 \notin C\left(V_{2}\right)$ by Fact 4.5 (2), and thus $|A|=|V(G)|-\left|V_{1}\right|-\left|V_{2}\right|-|B|-|C| \geq n-\left[g_{k-1}\left(K_{3}: F_{13}\right)-1\right]-\left[g r_{k-2}\left(K_{3}: F_{13}\right)-1\right]-1>3$. Then $1,2 \notin C(A)$, and so $|V(G)|=\left|V_{1}\right|+\left|V_{2}\right|+|A|+|B|+|C| \leq\left[g r_{k-1}\left(K_{3}: F_{13}\right)-1\right]+$ $2\left[g_{k-2}\left(K_{3}: F_{13}\right)-1\right]+1<n$, a contradiction. Therefore, $|B|=0$ and $|C| \leq 1$. Since $m \geq 4$, we have $|A| \geq 1$ and $|C|=1$. If $|A| \geq 3$, then $2 \notin C\left(V_{2}\right)$ and $1,2 \notin C(A)$. Thus $|V(G)| \leq\left[g r_{k-1}\left(K_{3}: F_{13}\right)-1\right]+2\left[g r_{k-2}\left(K_{3}: F_{13}\right)-1\right]+1<n$, a contradiction. If $|A|=2$, then $2 \notin C\left(V_{2}\right)$. Thus $|V(G)| \leq\left[g r_{k-1}\left(K_{3}: F_{13}\right)-1\right]+\left[g r_{k-2}\left(K_{3}: F_{13}\right)-1\right]+3<n$, a contradiction. Hence $|A|=|C|=1$. Then $|V(G)| \leq 2\left[g r_{k-1}\left(K_{3}: F_{13}\right)-1\right]+2<n$, a contradiction. 


\section{References}

[1] S.A. Burr and J.A. Roberts, On Ramsey numbers for stars, Utilitas Mathematica (1973), $217-220$.

[2] M. Chen, Y.S. Li and C.P. Pei, Gallai-Ramsey numbers of odd cycles and complete bipartite graphs, Graphs Combin. (2018). https://doi.org/10.1007/s00373-018-1931-7

[3] F.R.K. Chung and R. Graham, Edge-colored complete graphs with precisely colored subgraphs, Combinatorica 3 (1983), 315-324.

[4] R.J. Faudree, R. Gould, M. Jacobson and C. Magnant, Ramsey numbers in rainbow triangle free colorings, Australas. J. Combin. 46 (2010), 269-284.

[5] S. Fujita and C. Magnant, Gallai-Ramsey numbers for cycles, Discrete Math. 311 (2011), $1247-1254$.

[6] S. Fujita and C. Magnant, Extensions of Gallai-Ramsey results, J. Graph Theory 70 (2012), 404-426.

[7] S. Fujita, C. Magnant and K. Ozeki, Rainbow generalizations of Ramsey theory: a survey, Graphs Combin. 1 (2010), 1-30.

[8] S. Fujita, C. Magnant and K. Ozeki, Rainbow generalizations of Ramsey theory: a dynamic survey, Theo. Appl. Graphs 0 (2014), Article 1.

[9] T. Gallai, Transitiv orientierbare Graphen, Acta Math. Acad. Sci. Hungar 18 (1967), $25-66$.

[10] A. Gyárfás, G. N. Sárközy, A. Sebő and S. Selkow, Ramsey-type results for Gallai colorings, J. Graph Theory 64 (2010), 233-243.

[11] A. Gyárfás and G. Simonyi, Edge colorings of complete graphs without tricolored triangles, J. Graph Theory 46 (2004), 211-216.

[12] M. Hall, C. Magnant, K. Ozeki and M. Tsugaki, Improved upper bounds for GallaiRamsey numbers of paths and cycles, J. Graph Theory 75 (2014), 59-74.

[13] G.R.T. Hendry, Ramsey numbers for graphs with five vertices, J. Graph Theory 13 (1989), 245-248.

[14] F. Maffray and M. Preissmann, A translation of Tibor Gallai's paper: transitiv orientierbare Graphen, In: J.L. Ramirez-Alfonsin, B.A. Reed (Eds.), Perfect Graphs, Wiley, New York (2001), pp. 25-66.

[15] C. Magnant, Personal communication.

[16] Z.-X. Song and J.M. Zhang, A conjecture on Gallai-Ramsey numbers of even cycles and paths, arXiv:1803.07963 (2018).

[17] Z. Wang, Y.P. Mao, C. Magnant and J.Y. Zou, Ramsey and Gallai-Ramsey numbers for two classes of unicyclic graphs, arXiv:1809.10298 (2018).

[18] H.B. Wu, C. Magnant, P.S. Nowbandegani and S.M. Xia, All partitions have small partsGallai-Ramsey numbers of bipartite graphs, Discrete Appl. Math. in press (2018). 